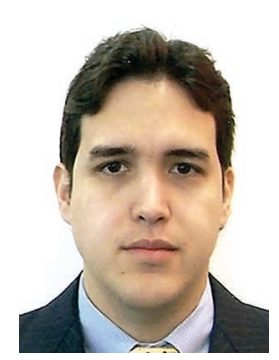

J. Romero, M.D.

\title{
CATHETER ABLATION FOR LONG-STANDING PERSISTENT ATRIAL FIBRILLATION
}

\author{
Jorge Romero, M.D. ${ }^{\text {a,b; }}$ Carola Gianni, M.D.ce; Luigi Di Biase, M.D., Ph.D. a,c,d,f; Andrea \\ Natale, M.D. ${ }^{\mathrm{c}, \mathrm{d}, \mathrm{g}, \mathrm{h}, \mathrm{i}, \mathrm{l}, \mathrm{I}}$ \\ aAlbert Einstein College of Medicine at Montefiore Hospital, Bronx, New York; ${ }^{b}$ Ronald Reagan UCLA Medical Center, \\ David Geffen School of Medicine at UCLA, Los Angeles, California; 'St. David's Medical Center, Austin, Texas; \\ ${ }^{d}$ University of Texas, Austin, Texas; 'University of Milan, Milan, Italy; 'University of Foggia, Foggia, Italy; ${ }^{\circ}$ California \\ Pacific Medical Center, San Francisco, California; 'hStanford University, Palo Alto, California; 'Case Western Reserve \\ University, Cleveland, Ohio; 'Scripps Clinic, San Diego, California
}

\begin{abstract}
Atrial fibrillation (AF) is the most common sustained arrhythmia worldwide and represents a major burden to health care systems. Atrial fibrillation is associated with a 4- to 5 -fold increased risk of thromboembolic stroke. The pulmonary veins have been identified as major sources of atrial triggers for AF. This is particularly true in patients with paroxysmal AF but not always the case for those with long-standing persistent AF (LSPAF), in which other locations for ectopic beats have been well recognized. Structures with foci triggering AF include the coronary sinus, the left atrial appendage (LAA), the superior vena cava, the crista terminalis, and the ligament of Marshall. More than 30 studies reporting results on radiofrequency ablation of LSPAF have been published to date. Most of these are observational studies with very different methodologies using different strategies. As a result, there has been remarkable variation in short- and long-term success, which suggests that the optimal ablation technique for LSPAF is still to be elucidated. In this review we discuss the different approaches to LSPAF catheter ablation, starting with pulmonary vein isolation (PVI) through ablation lines in different left atrial locations, the role of complex fractionated atrial electrograms, focal impulses and rotor modulation, autonomic modulation (ganglionated plexi), alcohol ablation, and the future of epicardial mapping and ablation for this arrhythmia. A stepwise ablation approach requires several key ablation techniques, such as meticulous PVI, linear ablation at the roof and mitral isthmus, electrogram-targeted ablation with particular attention to triggers in the coronary sinus and LAA, and discretionary right atrial ablation (superior vena cava, intercaval, or cavotricuspid isthmus lines).
\end{abstract}

\section{Introduction}

Atrial fibrillation (AF) is the most common sustained arrhythmia in the United States. In 2010, its prevalence ranged from 2.7 million to 6.1 million, and the projected number of individuals with $\mathrm{AF}$ is expected to rise to between 5.6 and 12 million by $2050 .{ }^{1}$ Atrial fibrillation is associated with a 4 - to 5 -fold increased risk of thromboembolic events (cerebrovascular accident, transient ischemic attack, and systemic emboli), and the percentage of strokes secondary to AF increases dramatically from $1.5 \%$ at 50 years of age to $23.5 \%$ at 80 years of age. ${ }^{2}$

The latest guidelines for the management of AF give a class $\mathrm{IIb}$ (Level of Evidence B) recommendation for catheter ablation of long-standing persistent AF (LSPAF), probably because of the low reported success rate in several studies and increased risk of complications when extensive radiofrequency ablation (RFA) is attempted. ${ }^{3}$ More than 30 studies reporting results on RFA of LSPAF have been published to date. Most of these are observational studies with very different methodologies using different strategies. As a result, there has been important variation in short- and long-term success rates using different techniques, which suggests that the optimal ablation technique for LSPAF is still to be elucidated. ${ }^{4}$

In this review we discuss the different approaches to LSPAF catheter ablation, starting from pulmonary vein isolation (PVI) through ablation lines in different left atrial (LA) locations, the role of complex fractionated atrial electrograms (CFAE), focal impulses, rotors and autonomic modulation, alcohol ablation, and the future of epicardial mapping and ablation for this arrhythmia.

\section{Mechanisms of Atrial Fibrillation}

Two main hypotheses, the multiple wavelet and mother rotor hypotheses, have been postulated as the main mechanisms of AF. According to the multiple wavelet hypothesis, originally proposed by Moe et al., the constant formation of new wavelets occurs through the process of wave splitting (wave-break), resulting in multiple wavelet fibrillation. ${ }^{5}$ The wave splitting occurs because of nonuniform dispersion of refractoriness and anatomic heterogeneity. This continuous propagation of multiple wavelets in the atria and wavelets as offspring of atrial reentry circuits might explain the mechanism by which AF is perpetuated without continuous focal discharge. Alternatively, Jalife et al. proposed the "mother rotor hypothesis," which establishes that AF is maintained by a single, stable, rapid periodic source (rapid reentrant circuit) of excitation that is unable to maintain 1:1 conduction throughout the myocardium. Each wavefront emerging from this mother rotor faces different functional and anatomical obstacles, leading to a wave break and ultimately to multiple independent wavelet formation. ${ }^{6}$ Interestingly, a human study demonstrated that both mechanisms can be present 
simultaneously and do not appear to be mutually exclusive, especially in the setting of persistent AF. ${ }^{7}$

In LSPAF, electrical and mechanical remodeling plays an important role in its perpetuation. Electrical remodeling, which is characterized by shortening of atrial refractoriness, develops within the first days of AF and contributes to stability of the arrhythmia. Nonetheless, AF maintenance must also depend on other mechanisms since the stability of AF continues to increase after electrical remodeling has been completed. Chronic atrial stretch induces activation of numerous signaling pathways leading to cellular hypertrophy, fibroblast proliferation, and tissue fibrosis. The resulting electroanatomical substrate is characterized by increased nonuniform anisotropy and local conduction heterogeneities facilitating reentry in the dilated atria. Moreover, atrial fibrosis may lead to disruption of the electrical side-to-side junctions between muscle fibers, which can result in electrical dissociation. ${ }^{8}$ As a result, mechanisms other than PV triggers are involved in the initiation and perpetuation of AF in patients with LSPAF, and a more extensive ablation, targeting additional targets such as complex-fractionated atrial electrograms (CFAE) and non PV-triggers, is often required.

\section{Reversible Causes}

The first step in managing LSPAF is having the patient undergo a detailed medical history and physical examination. Reversible or treatable causes of AF such as obesity, obstructive sleep apnea (OSA), uncontrolled hypertension, diabetes, hyperthyroidism, inflammatory and infectious diseases, metabolic syndrome, and stress may be triggering AF or contributing to its maintenance.

\section{Pulmonary Vein Isolation}

As demonstrated in the landmark study by Haïssaguerre et al., pulmonary veins (PV) are the main source of ectopic beats initiating paroxysmal AF. ${ }^{9}$ Special attention should be paid to obtain long-lasting isolation. In a study by Ouyang et al. examining patients with recurrent atrial tachycardias after PV isolation (PVI), approximately $80 \%$ of their PVs had reconnected..$^{10}$ PVI should always be confirmed by a circular mapping catheter demonstrating the absence or dissociation of PV potentials inside the ablation line. Wide antral PVI is more efficacious than ostial PVI in achieving freedom from any atrial tachyarrhythmia recurrence at long-term follow-up. ${ }^{11}$ Moreover, it has been shown that the antral region of the PVs includes considerable portions of the posterior LA wall down to the coronary sinus and to the left side of the septum. ${ }^{12}$ Isolating the LA posterior wall by creating superior and inferior lines between left and right PVs has a success rate ranging from $42 \%$ to $50 \%$ at 2 years. ${ }^{12-14}$

The contact force sensing catheter is a promising tool to improve the quality of ablation lesions when trying to achieve long-term complete PVI, as shown in the SMART-AF trial. Freedom from arrhythmia recurrence at 12 months was significantly higher ( $81 \%$ vs $66 \%$ ) for patients in whom stable contact force was applied during RFA. ${ }^{15}$

Nevertheless, despite a "perfect" PVI procedure, patients with LSPAF will require a more aggressive mapping and ablative approach. PVI is the most effective strategy for treating most patients with paroxysmal AF, but ablation strategies targeting PVI alone have consistently demonstrated a lower success rate in patients with persistent and LSPAF. Cheema et al. showed that single-procedure success performing PVI alone in the LSPAF population is as low as $20 \% .{ }^{16}$ Accordingly, it has been shown that PVs in LSPAF may play a passive role since either they are silent or their cycle length is greater than the LA cycle length. Also, some investigators have shown good AF success rates just by performing substrate ablation without touching PVs. ${ }^{17}$ Conversely, in persistent AF patients, between $40 \%$ and $60 \%$ experienced shortterm freedom from AF with just antral isolation.

\section{Complex Fractionated Atrial Electrograms}

Complex fractionated atrial electrograms (CFAEs) during $\mathrm{AF}$ are thought to represent either continuous reentry of the fibrillation waves into the restricted area or an overlap of different wavelets entering the same area at different times. ${ }^{18}$ These complex electrical activities have a relatively short cycle length and heterogeneous temporal and spatial distribution in humans. Ablation of these electrograms has been performed with the aim of eliminating wavelet reentry, thus preventing AF from perpetuating.

Nademanee et al. were the first to report the success of pure CFAE ablation. ${ }^{19}$ CFAEs were defined as (1) atrial electrograms that are fractionated and have two deflections or more over a 10 -second recording period, or (2) atrial electrograms with a very short cycle length $(\leq 120 \mathrm{~ms})$. They reported a single-procedure success rate of $63 \%$ at 12 months that improved to $77 \%$ with repeat procedures in 19 of the 64 patients. Nevertheless, similar results at that magnitude have not been replicated by other studies. In a randomized single-center study, for example, Oral et al. demonstrated that CFAE ablation in the left atrium was superior head to head to conventional PV and linear ablation. ${ }^{20}$

Subsequent studies analyzed the additive effect of CFAE ablation to PVI. Hayward et al. conducted a comprehensive metaanalysis of eight controlled studies comparing the effect of PVI with CFAE ablation versus PVI alone in patients with paroxysmal and non-paroxysmal AF $(\mathrm{N}=481) .{ }^{21}$ The authors found a slight benefit with the addition of CFAE ablation, with a relative risk of 1.15 (CI 1.2-1.31, $P=0.03$ ). This statistical difference was primarily driven by studies on patients with non-paroxysmal AF. Similar results were published in a meta-analysis by Kong et al., yet this group also analyzed the drawbacks of this technique and showed that adjunctive CFAE ablation increased procedural, fluoroscopy, and RFA times. ${ }^{22}$

\section{Linear Ablation}

Another approach is linear ablation, which compartmentalizes the atria with the aim of preventing the formation of macroreentrant circuits that have been postulated to maintain LSPAF. Ablation lines include a roof line, a mitral isthmus line, and an anterior line.

\section{Roof Line}

Hocini et al. was the first to describe the benefits of a complete linear block by creating a roof line joining the right and left superior PVs in patients with paroxysmal AF. ${ }^{23}$ This prospective randomized study demonstrated the feasibility of achieving complete linear block at the LA roof, which resulted in the prolongation of the fibrillatory cycle, termination of $\mathrm{AF}$, and subsequent noninducibility of this arrhythmia in the electrophysiology laboratory. Roof line was also associated with an improved clinical outcome compared with PVI alone (87\% vs 69\%, $P=0.04) .{ }^{39}$

\section{Mitral Line}

In a study by Jais et al., mitral isthmus block was achieved in $92 \%$ of patients. At 1 year after the last procedure, $87 \%$ patients 
with mitral isthmus ablation versus $69 \%$ without $(P=0.002)$ were arrhythmia free, with mitral isthmus ablation being the only factor associated with long-term success. ${ }^{24}$

\section{Anterior Lines}

Transection of the anterior LA is performed by delivering a roof line and then a perpendicular line that connects to the anterior mitral annulus. This approach is technically challenging, and complete transection results in significant delay in atrial activation with marked change in P-wave duration and morphology. Thus, this may result in the LA contracting after ventricular activation, potentially causing adverse atrial mechanical function and contributing to mechanical and electrical remodeling. ${ }^{25}$

A meta-analysis comparing the main six case series using PV antral isolation in conjunction with linear substrate modification reported a wide range of success, from $11 \%$ to $74 \% .4$ The highly variable results may indicate the significant different methodology and end points of each study as well as operator experience and ablation density. Whether defragmentation of these additional targets after PVI with linear lesions is superior to the actual ablation of non-PV triggers is also an open debate. Some studies have demonstrated that substrate modification with purely anatomical lesions, without any specific attempt to ablate trigger sites of AF, has been associated with higher recurrence rate. ${ }^{26}$

\section{Nonpulmonary Triggers for Atrial Fibrillation}

As described above, PVs have been identified as major sources of atrial triggers for AF. This is particularly true in patients with paroxysmal AF but not always the case for LSPAF, in which other locations for ectopic beats have been well recognized. Structures with foci triggering AF include the coronary sinus, the left atrial appendage (LAA), the superior vena cava, the crista terminalis, and the ligament of Marshall.

In general, ablation of AF triggers appears to be associated with higher success rates. ${ }^{27}$ Non-PV trigger ablation should be preferred to a blind substrate modification. ${ }^{28}$ Also, empirical ablation of sites associated with non-PV triggers such as the entire posterior wall, the roof, the anterior part of the LA septum, the LAA, the coronary sinus, and the superior vena cava has been shown to improve the freedom from AF at follow-up when combined with PV isolation. . $^{29,30}$

\section{The Left Atrial Appendage}

This structure deserves special consideration when managing patients with LSPAF. The arrhythmogenic role of the LAA is well known, and at least $30 \%$ of patients with persistent AF have LAA triggers being the only source of arrhythmia, with no PVs or other extrapulmonary vein site reconnection in $8.9 \%$ of the cases. ${ }^{31}$ When complete isolation of the LAA is achieved, only $15 \%$ of patients show recurrences compared to $74 \%$ in patients who do not receive ablation and $68 \%$ in patients who receive focal ablation of LAA triggers at 12 month follow-up. On the other hand, it might cause electromechanical dissociation with the potential for thrombus formation.

LAA can potentially be electrically isolated by surgical or transcatheter epicardial clipping occlusion. Starck et al. demonstrated complete electrical isolation of the LAA in 10 AF patients who underwent off-pump coronary artery bypass surgery with bilateral PV isolation and LAA clip occlusion. ${ }^{32}$ Han et al. studied 68 patients who underwent LAA ligation with the LARIAT® device (SentreHEART, Inc., Redwood City, CA) and verified a significant decrease in LAA voltages before and after the snare was tightened, with complete isolation of the appendage evident in $90 \%$ of patients. ${ }^{33}$ Lakkireddy et al. conducted a prospective observational study to estimate the benefit of concomitant LARIAT procedure when added to conventional AF ablation. The primary outcome of AF recurrence at 1-year followup was lower in the LARIAT group ( $34 \%$ vs $55 \%, P=0.025)$ after the first ablation. Also, fewer patients in the LARIAT group needed repeat ablation $(15 \%$ vs $35 \%, P=0.04) .{ }^{34}$ The incremental benefit of decreasing the recurrence of AF after ablation by the LARIAT device has to be assessed by randomized clinical trials.

\section{Focal Impulse and Rotor Modulation}

Narayan et al. hypothesized that human AF may be sustained by localized sources such as electrical rotors and focal impulses. In the CONFIRM trial, these sources were detected in $97 \%$ of 107 patients undergoing paroxysmal or persistent AF ablation. ${ }^{35}$ The acute end point (AF termination or consistent slowing) was achieved in $86 \%$ of cases guided by focal impulse and rotor modulation (FIRM) versus $20 \%$ of conventional ablation cases $(P<$ 0.001). During a median follow-up of 273 days following a single procedure, FIRM-guided cases had higher freedom from AF $(82.4 \%$ vs $44.9 \% ; P<0.001)$.

Interestingly, a different group from Taiwan using a distinctive optical mapping system also demonstrated the possibility of localizing rotors in the LA in a canine heart failure model in which AF was induced by infusing acetylcholine. Epicardial ablation of the rotor anchoring sites suppressed AF inducibility in 12 out of 13 Langendorff-perfused left PV-LA preparations. ${ }^{36}$

The use of rotor mapping and ablation should be considered investigational, and a large, multicenter, randomized controlled trial comparing FIRM versus conventional ablation versus FIRM and PVI must be designed and conducted to validate the efficacy and safety of this technique.

\section{Autonomic Modulation}

The autonomic system activity plays a very important role in the initiation and maintenance of $\mathrm{AF}$, and modulating autonomic nerve function may contribute to AF control using potential therapeutic applications such as ganglionated plexi (GP) ablation, renal sympathetic denervation, cervical vagal nerve stimulation, baroreflex stimulation, and cutaneous stimulation, among others.

Left atrial GPs are part of the intrinsic cardiac autonomic nervous system and have been implicated in the pathogenesis of AF. Ganglionated plexi sites identified using continuous highfrequency stimulation before antral PVI or circumferential PV ablation were almost entirely absent on retesting after ablation. ${ }^{37}$ Katritsis et al. conducted the first randomized clinical trial evaluating autonomic denervation added to PVI in 242 patients with paroxysmal AF. ${ }^{63}$ The investigators demonstrated that pairing GP ablation with PVI confers a significantly higher success rate compared to either PVI or GP alone at 2-year follow-up using implantable loop recorders. ${ }^{38}$ Nevertheless, this approach has not been widely implemented since animal studies have shown that ablation of these GP may have paradoxical proarrhythmic effects increasing the AF burden and AF inducibility. ${ }^{39}$

\section{Alcohol Ablation}

The ligament of Marshall is associated with triggers initiating AF. It usually is ablated from the endocardial aspect of the left atrium inferior to the ostium of the left inferior pulmonary vein, although it may occasionally require direct catheterization from within the coronary sinus. Recently, ethanol infusion in the vein of 


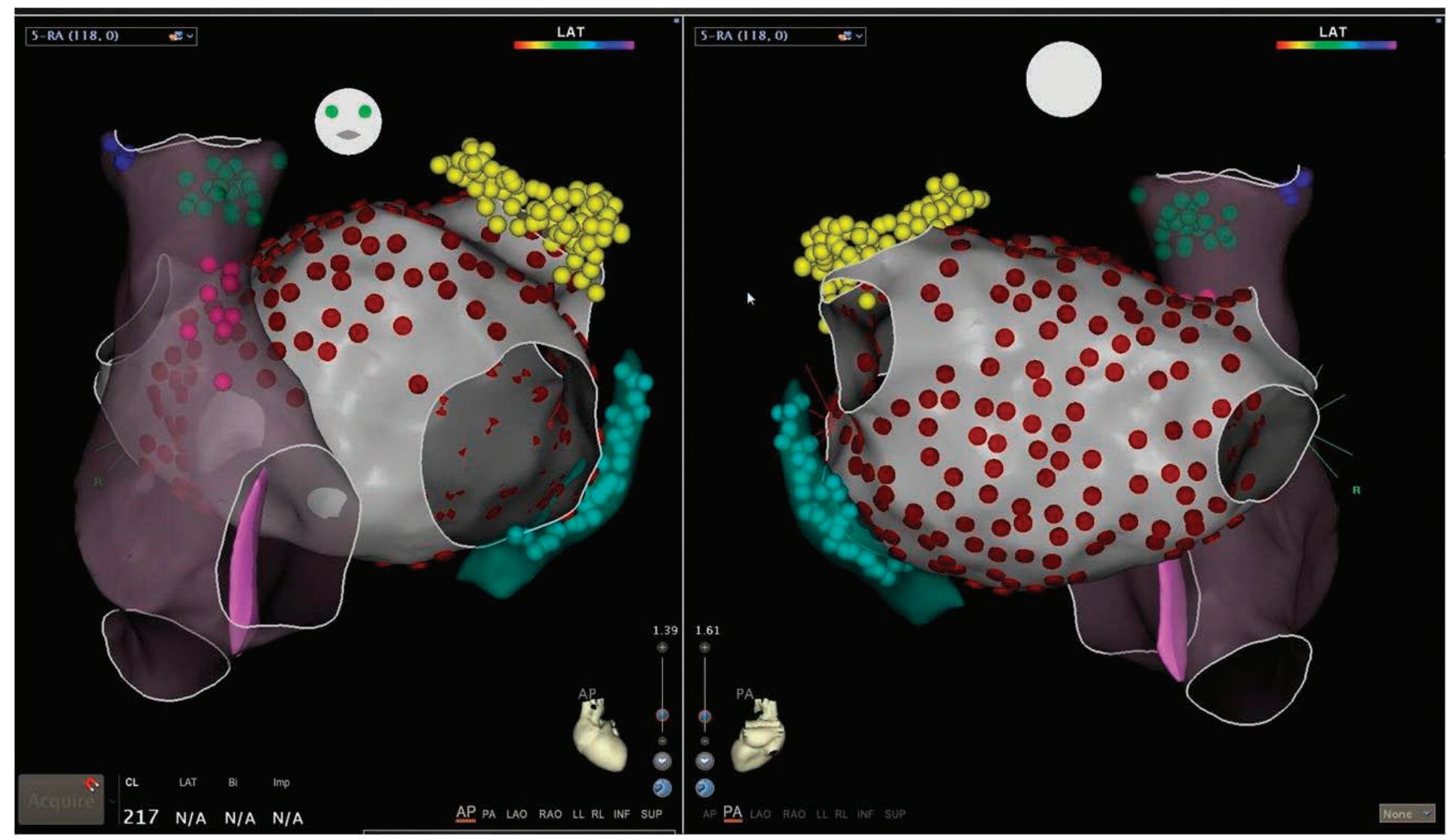

Figure 1. Anteroposterior (AP, left panel) and posteroanterior (PA, right panel) views of a 3-dimenstional electroanatomic map of the left atrium. The patient, who has long-standing persistent atrial fibrillation, underwent extensive ablation with pulmonary vein (PV) antrum and posterior wall isolation (red arrows) plus ablation of non-PV triggers such as the left atrial appendage (yellow dots) the coronary sinus (light green dots) and the superior vena cava (green dots).

Marshall was shown to achieve effective ablation of this structure throughout its course between the mitral annulus and the left superior PV. ${ }^{40}$ Of note, ethanol infusion in the vein of Marshall was associated with effective isolation of the left inferior PV in $40 \%$ of patients. The clinical relevance of ligament of Marshall ablation is still undefined and warrants investigation in further studies.

\section{Epicardium}

Recent mapping studies in goats with persistent AF showed that electrical dissociation in the LA occurs not only between neighboring muscle bundles but also in the third dimension (i.e., between the epicardial layer and the endocardial bundle network) Such endo-epicardial dissociation will significantly increase the number of wavefronts that can simultaneously be present in the atrial wall. ${ }^{41}$ Moreover, the electrophysiological characteristics of the atrial epicardium are different from the endocardium, with the former having a shorter effective refractory period and more frequent atrial repetitive response induction by programmed stimulation. ${ }^{42} \mathrm{~A}$ recent study by Lee et al. performing high-density epicardial mapping of the posterior LA wall, LAA, and right superior PV-LA junction in 18 patients with LSPAF showed that this arrhythmia was highly dynamic and had heterogeneous patterns of atrial activation consisting predominantly of multiple unstable wavefronts and disorganized activity. ${ }^{43}$

\section{Surgery}

In 2012, Boersma et al. published the first randomized clinical trial comparing video-assisted thoracoscopy surgical ablation (PVI, GP ablation, and LAA excision with optional additional lines) versus catheter ablation (linear antral PVI and optional additional lines) ${ }^{44}$ Although the study demonstrated significant AF-free survival in the surgical group at 1-year follow-up (65.6\% vs $36 \%$ ), the primary safety end point of significant adverse events during the 12-month follow-up was significantly higher for the surgical ablation group ( $34.4 \%$ vs $15.9 \%$ ). These adverse events were mainly determined by procedural complications such as pneumothorax, major bleeding, and the need for permanent pacemaker. It is worth mentioning that these two groups are difficult to compare due primarily to the fact that the catheter ablation group did not undergo LAA isolation and GP ablation, and specific ablation lesions were not prespecified for both arms of the study.

\section{Recommended Approach}

A stepwise ablation approach requires several key ablation techniques, such as meticulous PVI, linear ablation at the roof and mitral isthmus, electrogram-targeted ablation with particular attention to triggers in the coronary sinus and LAA, and discretionary right atrial ablation (superior vena cava, intercaval, or cavotricuspid isthmus lines). This approach is associated with a single-procedure success rate that has ranged from $38 \%$ to $62 \%$ at approximately 18 -months follow-up. The integration of repeat procedures, mostly for focal atrial tachycardia and flutter, increased the clinical success rate to between $70 \%$ and $88 \%{ }^{45-47}$

The circular mapping catheter should be positioned under fluoroscopic guidance and confirmed by intracardiac echocardiogram at the antrum of each PV. Pulmonary vein potentials recorded by the circular mapping catheter are the 


\section{Long-standing Persistent AF Ablation Outcomes Short- and Long-Term Success}

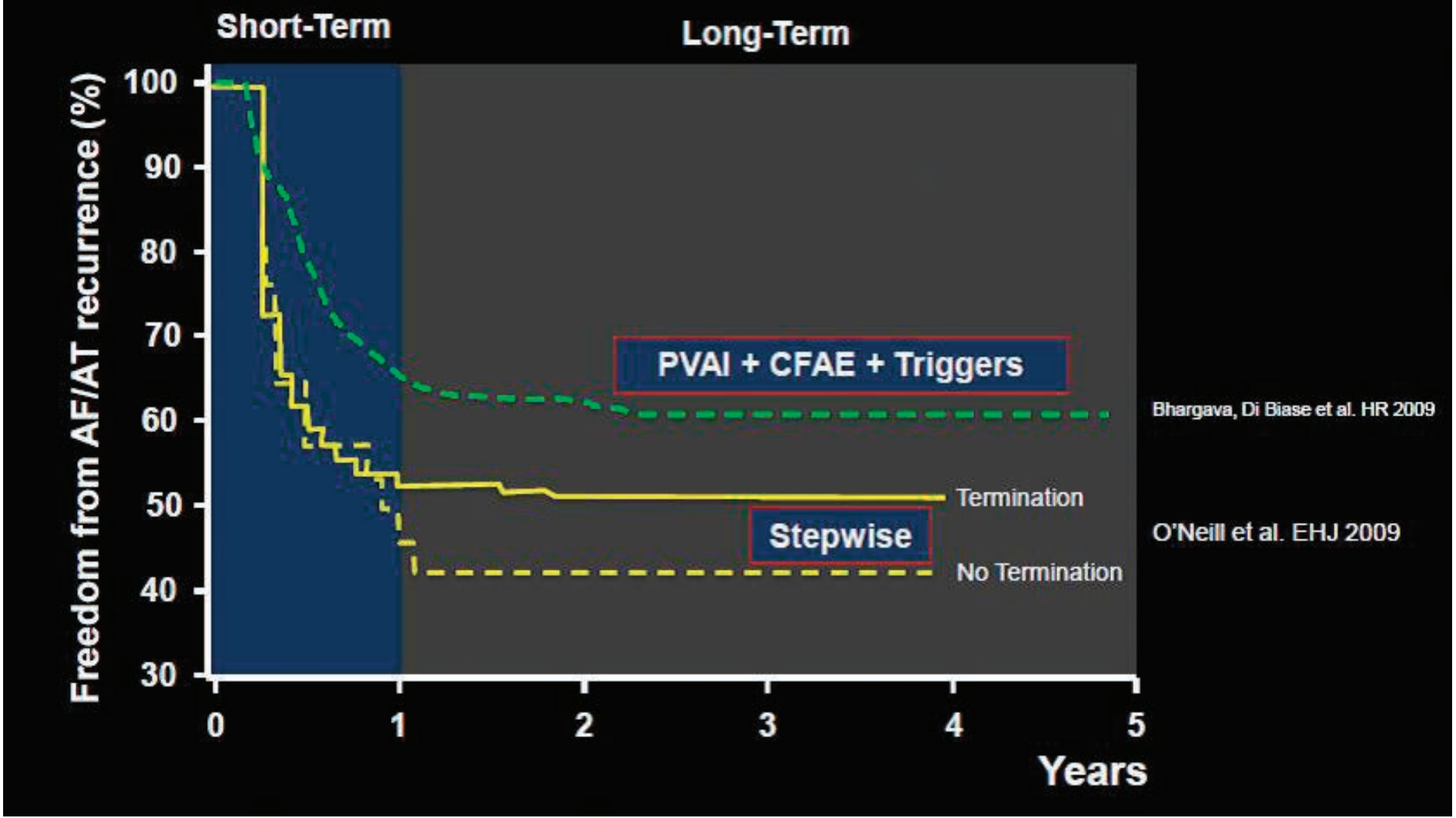

Figure 2. Different success rates achieved by the stepwise approach versus a similar strategy, with elimination of non-pulmonary vein trigger. Possible reasons for the higher success rate with the triggers approach may include a more proximal left atrial isolation, systematic isolation of the superior vena cava, and emphasis given to sustained and non-sustained non-PV trigger ablation.

target for ablation until abolition of all PV antrum electrograms is achieved. The left PV are usually isolated first, then the ablation encompasses the posterior wall contained within the left and right $\mathrm{PV}$, and consequently the right PVs are isolated. Entrance and exit block is verified. The electrical isolation of the PVs is extended to the entire posterior wall down to the coronary sinus and to the left side of the septum. Ablation of CFAEs in the left atrium and coronary sinus is also performed if fractionated potentials are found. Finally, identification of potential triggers is attempted, including careful and complete LAA isolation (Figure 1).

If termination cannot be achieved after extensive ablation, cardioversion is performed. Procedural termination of AF is not a reliable end point. Indeed, two separate studies found that patients who had termination/organization of AF versus those who required cardioversion showed no difference in terms of arrhythmia recurrence, probably because patients in whom AF was terminated had a higher incidence of atrial tachycardia following ablation. ${ }^{48,49}$ Moreover, seeking AF termination may increase procedural/fluoroscopy time and complications. ${ }^{49}$ (Figure 2).

After stable sinus rhythm is achieved, isoproterenol (up to 30 $\mathrm{mcg} / \mathrm{min}$ for 15 to $20 \mathrm{~min}$ ) is administered to disclose any non-
PV triggers or tachycardia and to look for acute PV reconnection. Adenosine administration has also been recommended. Although the mechanism by which adenosine enhances conduction or triggers AF is not totally understood, this phenomenon is thought to be mediated by adenosine's effects on shortening atrial action potential duration and refractoriness. Because adenosine has little effect on atrial conduction velocity, the net effect of adenosine is to shorten the wavelength of activation, thereby potentiating AF. Adenosine can also hyperpolarize dormant PV myocytes, increasing excitability, and thus triggering PV ectopy. ${ }^{50}$

\section{Conclusion}

Catheter ablation for rhythm control in patients with LSPAF remains challenging. No single strategy is consistently effective in this population. Pulmonary vein and posterior wall isolation are essential steps, but identification and ablation of non-PV triggers is important to achieve long-term success, and substrate modification should be sought in every patient. Moreover, outcomes improve after repeat procedures. The importance of extending the ablation to non-PV sites is supported by clinical evidence and related to the significant structural and electrophysiological changes that take 
place in LSPAF, known as "atrial remodeling." New techniques and targets currently under investigation could prove important in helping achieve rhythm control, but longer randomized controlled trials are needed to evaluate their long-term effectiveness and safety.

Conflict of Interest Disclosure: Dr. Di Biase is a consultant for Biosense Webster Inc., St. Jude Medical, Boston Scientific Corp., EpiEP, Inc., and Biotronik; Dr. Natalie is a consultant for Janssen Pharmaceuticals, Biosense Webster Inc., St. Jude Medical, Boston Scientific Corp., and Medtronic.

Keywords: atrial fibrillation; catheter ablation; pulmonary vein isolation, longstanding persistent atrial fibrillation, complex fractionated atrial electrograms, linear ablation

\section{References}

1. Miyasaka Y, Barnes ME, Gersh BJ, et al. Secular trends in incidence of atrial fibrillation in Olmsted County, Minnesota, 1980 to 2000, and implications on the projections for future prevalence. Circulation. 2006 Jul 11;114(2):119-25.

2. Wolf PA, Abbott RD, Kannel WB. Atrial fibrillation as an independent risk factor for stroke: the Framingham Study. Stroke. 1991 Aug;22(8):983-8.

3. January CT, Wann LS, Alpert JS, et al. 2014 AHA/ACC/HRS guideline for the management of patients with atrial fibrillation: a report of the American College of Cardiology/American Heart Association Task Force on practice guidelines and the Heart Rhythm Society. Circulation. 2014 Dec 2;130(23):e199-267.

4. Brooks AG, Stiles MK, Laborderie J, et al. Outcomes of longstanding persistent atrial fibrillation ablation: a systematic review. Heart Rhythm. 2010 Jun;7(6):835-46.

5. Moe GK, Abildskov JA. Atrial fibrillation as a self-sustaining arrhythmia independent of focal discharge. Am Heart J. 1959 Jul;58(1):59-70.

6. Jalife, J, Berenfeld O, Skanes A, Mandapati R. Mechanisms of atrial fibrillation: mother rotors or multiple daughter wavelets, or both? J Cardiovasc Electrophysiol. 1998 Aug;9(8 Suppl):S2-12.

7. Konings KT, Kirchhof CJ, Smeets JR, Wellens HJ, Penn OC, Allessie MA. High-density mapping of electrically induced atrial fibrillation in humans. Circulation. 1994 Apr;89(4);1665-80.

8. Eckstein J, Verheule S, de Groot NM, Allessie M, Schotten $\cup$. Mechanisms of perpetuation of atrial fibrillation in chronically dilated atria. Prog Biophys Mol Biol. 2008 JunJul;97(2-3):435-51.

9. Haïssaguerre M, Jaïs P, Shah DC, et al. Spontaneous initiation of atrial fibrillation by ectopic beats originating in the pulmonary veins. N Engl J Med. 1998 Sep 3;339(10):659-66.

10. Ouyang F, Antz M, Ernst S, et al. Recovered pulmonary vein conduction as a dominant factor for recurrent atrial tachyarrhythmias after complete circular isolation of the pulmonary veins: lessons from double Lasso technique. Circulation. 2005 Jan 18;111(2):127-35.

11. Proietti R, Santangeli P, Di Biase L, et al. Comparative effectiveness of wide antral versus ostial pulmonary vein isolation: a systematic review and meta-analysis. Circ Arrhythm Electrophysiol. 2014 Feb;7(1):39-45.

12. Kanj M, Wazni $O$, Natale A. Pulmonary vein antrum isolation. Heart Rhythm. 2007 Mar;4(3 Suppl):S73-9.

13. Sanders $P$, Hocini $M$, Jaiis $P$, et al. Complete isolation of the pulmonary veins and posterior left atrium in chronic atrial fibrillation. Long-term clinical outcome. Eur Heart J. 2007 Aug;28(15):1862-71.

14. Chen J, Off MK, Solheim E, Schuster P, Hoff PI, Ohm OJ. Treatment of atrial fibrillation by silencing electrical activity in the posterior inter-pulmonary-vein atrium. Europace. 2008 Mar;10(3):265-72.

15. Natale A, Reddy VY, Monir G, et al. Paroxysmal AF catheter ablation with a contact force sensing catheter: results of the prospective, multicenter SMART-AF trial. J Am Coll Cardiol. 2014 Aug 19;64(7):647-56.

16. Cheema A, Dong J, Dalal D, et al. Circumferential ablation with pulmonary vein isolation in permanent atrial fibrillation. Am J Cardiol. 2007 May 15;99(10):1425-8.

17. Seitz J, Horvilleur J, Curel L, et al. Active or passive pulmonary vein in atrial fibrillation: is pulmonary vein isolation always essential? Heart Rhythm. 2014 Apr;11(4):579-86.

18. Konings KT, Kirchhof CJ, Smeets JR, Wellens HJ, Penn OC, Allessie MA. High-density mapping of electrically induced atrial fibrillation in humans. Circulation. 1994 Apr;89(4):1665-8.

19. Nademanee K, McKenzie J, Kosar E, et al. A new approach for catheter ablation of atrial fibrillation: mapping of the electrophysiologic substrate. J Am Coll Cardiol. 2004 Jun 2;43(11):2044-53.

20. Oral H, Chugh A, Good E, et al. Randomized evaluation of right atrial ablation after left atrial ablation of complex fractionated atrial electrograms for long-lasting persistent atrial fibrillation. Circ Arrhythm Electrophysiol. 2008 Apr;1(1):6-13.

21. Hayward RM, Upadhyay GA, Mela T, et al. Pulmonary vein isolation with complex fractionated atrial electrogram ablation for paroxysmal and nonparoxysmal atrial fibrillation: A meta-analysis. Heart Rhythm. 2011 Jul;8(7):994-1000.

22. Kong MH, Piccini JP, Bahnson TD, et al. Efficacy of adjunctive ablation of complex fractionated atrial electrograms and pulmonary vein isolation for the treatment of atrial fibrillation: a meta-analysis of randomized controlled trials. Europace. 2011 Feb;13(2):193-204.

23. Hocini M, Jaïs $P$, Sanders $P$, et al. Techniques, evaluation, and consequences of linear block at the left atrial roof in paroxysmal atrial fibrillation: a prospective randomized study. Circulation. 2005 Dec 13;112(24):3688-96.

24. Jaïs $P$, Hocini M, Hsu LF, et al. Technique and results of linear ablation at the mitral isthmus. Circulation. 2004 Nov 9;110(19):2996-3002.

25. Sanders P, Jaïs P, Hocini M, et al. Electrophysiologic and clinical consequences of linear catheter ablation to transect the anterior left atrium in patients with atrial fibrillation. Heart Rhythm. 2004 Jul;1(2):176-184.

26. Garg, A, Finneran W, Mollerus M, et al. Right atrial compartmentalization using radiofrequency catheter ablation for management of patients with refractory atrial fibrillation. $J$ Cardiovasc Electrophysiol. 1999 Jun;10(6):763-71.

27. Di Biase L, Santangeli P, Natale A. How to ablate long-standing persistent atrial fibrillation? Curr Opin Cardiol. 2013 Jan;28(1):2635.

28. Gerstenfeld EP, Callans DJ, Dixit S, Zado E, Marchlinski FE. Incidence and location of focal atrial fibrillation triggers in patients undergoing repeat pulmonary vein isolation: implications for ablation strategies. J Cardiovasc Electrophysiol. 2003 Jul;14(7):685-90.

29. Lin WS, Tai CT, Hsieh MH, et al. Catheter ablation of paroxysmal atrial fibrillation initiated by non-pulmonary vein ectopy. Circulation. 2003 Jul 1;107(25):3176-83.

30. Arruda M, Mlcochova H, Prasad SK, et al. Electrical isolation of the superior vena cava: an adjunctive strategy to pulmonary vein antrum isolation improving the outcome of AF ablation. $J$ Cardiovasc Electrophysiol. 2007 Dec;18(12):1261-6. 
31. Di Biase L, Burkhardt JD, Mohanty P, et al. Left atrial appendage: an underrecognized trigger site of atrial fibrillation. Circulation. 2010 Jul 13;122(2):109-18.

32. Starck, CT, Steffel J, Emmert MY, et al. Epicardial left atrial appendage clip occlusion also provides the electrical isolation of the left atrial appendage. Interact Cardiovasc Thorac Surg. 2012 Sep;15(3):416-8.

33. Han FT, Bartus K, Lakkireddy D, et al. The effects of LAA ligation on LAA electrical activity. Heart Rhythm. 2014 May;11(5):864-70.

34. Lakkireddy D, Reddy M, ARM Sridhar, et al. Left atrial appendage ligation and ablation for persistent atrial fibrillation (LAALA-AF Registry). J Am Coll Cardiol. 2014 Apr 1;63(12S):A390.

35. Narayan SM, Krummen DE, Shivkumar K, Clopton P, Rappel WJ, Miller JM. Treatment of atrial fibrillation by the ablation of localized sources: CONFIRM (Conventional Ablation for Atrial Fibrillation With or Without Focal Impulse and Rotor Modulation) trial. J Am Coll Cardiol. 2012 Aug 14;60(7):628-36.

36. Chou CC, Chang PC, Wen MS, et al. Epicardial ablation of rotors suppresses inducibility of acetylcholine-induced atrial fibrillation in left pulmonary vein-left atrium preparations in a beagle heart failure model. J Am Coll Cardiol. 2011 Jul 5;58(2):158-66.

37. Verma A, Saliba WI, Lakkireddy D, et al. Vagal responses induced by endocardial left atrial autonomic ganglion stimulation before and after pulmonary vein antrum isolation for atrial fibrillation. Heart Rhythm. 2007 Sep;4(9):1177-82.

38. Katritsis DG, Pokushalov E, Romanov A, et al. Autonomic denervation added to pulmonary vein isolation for paroxysmal atrial fibrillation: a randomized clinical trial. J Am Coll Cardiol. 2013 Dec 17;62(24):2318-25.

39. Lo LW, Scherlag BJ, Chang HY, Lin YJ, Chen SA, Po SS. Paradoxical long-term proarrhythmic effects after ablating the "head station" ganglionated plexi of the vagal innervation to the heart. Heart Rhythm. 2013 May;10(5):751-7.

40. Dave AS, Báez-Escudero JL, Sasaridis C, Hong TE, Rami T, Valderrábano M. Role of the vein of Marshall in atrial fibrillation recurrences after catheter ablation: therapeutic effect of ethanol infusion. J Cardiovasc Electrophysiol. 2012 Jun;23(6):583-91.
41. Verheule S, Eckstein J, Linz D, et al. Role of endo-epicardial dissociation of electrical activity and transmural conduction in the development of persistent atrial fibrillation. Prog Biophys Mol Biol. 2014 Aug;115(2-3):173-85.

42. Michowitz Y, Nakahara S, Bourke T, et al. Electrophysiological differences between the epicardium and the endocardium of the left atrium. Pacing Clin Electrophysiol. 2011 Jan;34(1):37-46.

43. Lee G, Kumar S, Teh A, et al. Epicardial wave mapping in human long-lasting persistent atrial fibrillation: transient rotational circuits, complex wavefronts, and disorganized activity. Eur Heart J. 2014 Jan;35(2):86-97.

44. Boersma LV, Castella M, van Boven W, et al. Atrial fibrillation catheter ablation versus surgical ablation treatment (FAST): a 2-center randomized clinical trial. Circulation. 2012 Jan 3;125(1):23-30.

45. Takahashi Y, O'Neill MD, Hocini M, et al. Characterization of electrograms associated with termination of chronic atrial fibrillation by catheter ablation. J Am Coll Cardiol. 2008 Mar 11;51(10):1003-10.

46. Rostock T, Steven D, Hoffmann B, et al. Chronic atrial fibrillation is a biatrial arrhythmia: data from catheter ablation of chronic atrial fibrillation aiming arrhythmia termination using a sequential ablation approach. Circ Arrhythm Electrophysiol. 2008 Dec;1(5):344-53.

47. Haïssaguerre M, Hocini M, Sanders P, et al. Catheter ablation of long-lasting persistent atrial fibrillation: clinical outcome and mechanisms of subsequent arrhythmias. J Cardiovasc Electrophysiol. 2005 Nov; 16(11):1138-47.

48. O'Neill MD, Wright M, Knecht S, et al. Long-term follow-up of persistent atrial fibrillation ablation using termination as a procedural endpoint. Eur Heart J. 2009 May;30(9):1105-12.

49. Elayi CS, Di Biase L, Barrett C, et al. Atrial fibrillation termination as a procedural endpoint during ablation in long-standing persistent atrial fibrillation. Heart Rhythm. 2010 Sep;7(9):121623.

50. Ip JE, Cheung JW, Chung JH, et al. Adenosine-induced atrial fibrillation: insights into mechanism. Circ Arrhythm Electrophysiol. 2013 Jun;6(3):e34-7. 